\title{
Adjoint-BASED Estimation AND CONTROL of SPATIAL, TEMPoral AND STOCHASTIC APPROXIMATION ERRORS IN UNSTEADY FLOW SIMULATIONS
}

\author{
VINOD K. LAKSHMINARAYAN ${ }^{\mathrm{A}, 1, *}$, KARTHIK DURAISAMY ${ }^{\mathrm{B}, 2}$ \\ ${ }^{a}$ Science and Technology Corporation, NASA Ames Research Center, Moffett Field, CA 94035, USA \\ ${ }^{b}$ Department of Aerospace Engineering, University of Michigan, Ann Arbor, MI 48109, USA
}

\begin{abstract}
The ability to estimate various sources of numerical error and to adaptively control them is a powerful tool in quantifying uncertainty in predictive simulations. This work attempts to develop reliable estimates of numerical errors resulting from spatial, temporal and stochastic approximations of fluid dynamic equations using a discrete adjoint approach. Each of these sources of error are isolated into distinct components and the accuracy of their estimation is verified. When applied to unsteady flow simulations of vertical axis wind turbines (VAWT), the procedure demonstrates good recovery of discretization errors to provide accurate estimate of the objective functional. The framework is then applied to a VAWT simulation with inherent stochasticity and is confirmed to effectively estimate errors in computing statistical quantities of interest. The ability to use these stochastic error estimates as a basis for adaptive sampling is also presented. Predictive science is typically constrained by finite computational resources and this work demonstrates the viability of adjoint-based approaches to budget available computational resources to effectively pursue uncertainty quantification.
\end{abstract}

Keywords: error estimation, discrete adjoints, uncertainty quantification, vertical axis wind turbine

\section{Introduction}

Predictive simulations are increasingly being employed in scientific applications and effective use of these tools is a balancing act between available computational resources and the desired numerical accuracy. For the computations to be of value, it is critical to ensure that numerical errors are below a threshold such that the results of the simulation can be confidently used in analysis and design. An estimate of various sources of numerical error can not only provide a powerful tool in formal verification of the computational simulation, but also help in budgeting the available resources towards minimizing these errors.

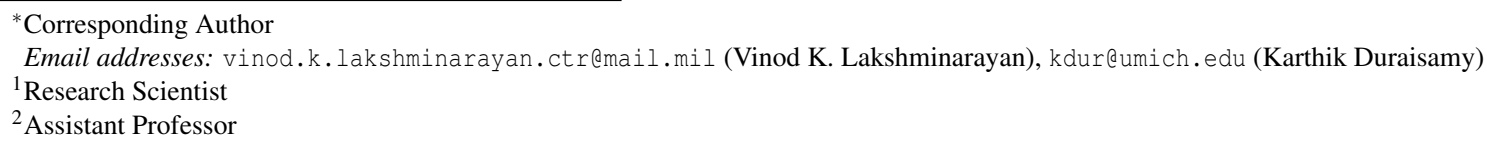


Approaches to quantifying numerical error in the context of finite elements have been pursued for the past two decades $[1,2,3]$, primarily with the objective of providing an indicator of the local contribution to the functional error. Pierce and Giles [4] presented a generic framework applicable to finite element/volume/difference based discretizations, that demonstrated super-convergent functional estimates by adding a correction term based on the adjoint (or a dual) of the original governing equations. Venditti and Darmofal [5] proposed an algebraic equivalent of the Pierce and Giles [4] approach. This approach utilizes the discrete adjoint equations and the functional error on the baseline mesh is improved by computing an estimate of the functional on a refined mesh. More recently, several researches have used similar approaches for error estimation and control in finite volume framework [6, 7]. A comprehensive literature review on output-based error estimation is provided by Fidkowski and Darmofal [8]. The current work pursues the approach of Venditti and Darmofal [5] because the discrete formulation allows for a natural extension to account for temporal and stochastic errors. In the past, the current authors have employed adjoint methods in the areas of error-estimation/control [9, 10] and uncertainty quantification [10, 11, 12, 13].

Many unsteady fluid and aerodynamic problems can be approximated using the assumption of periodicity in time. In solving such periodic problems, the time-spectral method $[14,15]$ has proved to be highly efficient. The basic idea of the time-spectral method is to have a Fourier representation of the time-derivative term of the unsteady flow equation to take advantage of the periodicity. When transformed back to the physical domain, the time derivative term appears as a high-order finite difference formula coupling all the time levels. The solution can then be obtained by marching towards a steady-state in an auxiliary pseudo-time variable. The time-spectral method is similar in spirit to the frequency-domain method (e.g. Hall et al. [16]), but is different in the sense that the computations are performed directly in the time domain instead of the frequency domain. One of the major advantages of the time-spectral method is that it makes the adjoint method more affordable in unsteady flow simulations as the requirement of saving and working on the solution at every time-step can be avoided.

In this work a vertical axis wind turbine (VAWT) problem, that has periodic solution in time is pursued as a sample application. The highly unsteady nature of the flow-field seen in these calculations present many challenges to spatial and temporal approximations. Further, wind turbine simulations are subject to a range of uncertainties, arising either from natural variabilities present in the system such as the physical variation in wind speed and free stream turbulence. Quantifying the impact of such uncertainties can improve the computational predictions and can aid in design of cost-effective wind turbines. In addition, in many engineering applications, one is typically interested in statistical moments like the mean and variance of some functional (e.g. power generated by the wind turbine) in the stochastic space. For reliability, it is necessary to ensure that the numerical error in calculating these statistical moments are estimated and controlled. 
Duraisamy and Chandrashekar [12] proposed a framework based on the use of adjoint equations to formulate an adaptive sampling strategy for uncertainty quantification for problems governed by algebraic or differential equations involving random parameters. The approach makes use of discrete sampling based on collocation on simplex elements [17] in stochastic space. Errors resulting from inexact reconstruction of the solution within the simplex elements are estimated. This framework is adopted in the present work and is extended to highly unsteady and non-linear problems.

The primary objective of this paper is to carefully evaluate the aforementioned adjoint-based error estimation strategies in spatial, temporal and stochastic approximations in practical aerodynamic applications. The main problem considered is a vertical axis wind turbine case. This setup is chosen as it can be simulated in two spatial dimensions, thus allowing for a feasible problem but without oversimplifying the flow-field. The following sections contain the formulations required for calculation of each component of the error estimates, implementation details and results showing the benefits of such estimates to help budget computational resources as well as to provide a basis for adaptive sampling in stochastic space.

\section{Governing Equations of Fluid Flow}

Let $\Omega_{s} \subset \mathbb{R}^{3}$ denote the fluid domain of interest and $\Omega_{h_{s}}$ its discretization with $N_{x}, N_{y}$ and $N_{z}$ partitions in the three spatial directions $x, y$ and $z$, respectively. The Navier-Stokes equations in a semi-discrete form can be written as

$$
\frac{\partial U(\vec{x}, t)}{\partial t}+\hat{R}(U(\vec{x}, t))=0, \quad \vec{x} \in \Omega_{h_{s}}
$$

where $U(\vec{x}, t)$ represents the approximation of the state vector of unknowns in the semi-discrete space and $\hat{R}(U(\vec{x}, t))$ is the residual of spatial discretization of inviscid and viscous fluxes including the grid velocity terms to account for mesh motion. For problems that are periodic in time, the Time Spectral method[14] can be employed as an efficient alternative to traditional time marching methods. In this approach, a Fourier representation is utilized in the time domain, $\Omega_{t}$. If the time period $T$ is divided into $N_{t}$ time intervals, the time derivative term can be written as a matrixvector product, $D_{t} U(\vec{r}) . U(\vec{r})$ denotes the discrete representation of the state vector of unknowns containing the solution state at all $N_{t}$ time instances and $\vec{r} \in \Omega_{h_{s}} \cup \Omega_{h_{t}}$, where $\Omega_{h_{t}}$ is the discrete representation of $\Omega_{t}$. $D_{t}$ is a matrix whose elements for odd and even $N_{t}$ 's are given in Ref. 14. 


$$
\begin{aligned}
& d_{i j}^{\text {odd }}= \begin{cases}\frac{\pi}{T}(-1)^{l-j} \operatorname{cosec}\left(\frac{\pi(l-j)}{N_{t}}\right) & : l \neq j \\
0 & : l=j\end{cases} \\
& d_{i j}^{\text {even }}= \begin{cases}\frac{\pi}{T}(-1)^{l-j} \cot \left(\frac{\pi(l-j)}{N_{t}}\right) & : l \neq j \\
0 & : l=j\end{cases}
\end{aligned}
$$

The governing equation can now be written as

$$
D_{t} U(\vec{r})+\hat{R}(U(\vec{r}))=0
$$

Combining the time derivative term with the residual term, the system of discrete equations can be written in a compact form as

$$
R(U(\vec{r}))=0
$$

The above governing equation in the presence of random parameters $\vec{\xi}$ can be written as

$$
R(U(\vec{r}, \vec{\xi}))=0, \quad \vec{\xi} \in \Omega_{h_{\xi}},
$$

where $\Omega_{h_{\xi}}$ is the discrete representation of the stochastic space, $\Omega_{\xi} \subset \mathbb{R}^{n_{\xi}}, n_{\xi}$ is the number of stochastic variables. $\Omega_{h_{\xi}}$ can be discretized into $N_{E}$ simplex elements consisting of $N_{S}$ vertices.

\section{Flow Solution Procedure}

In the current work, the spatial discretization is evaluated using a cell-centered finite volume formulation on structured grids. The inviscid Euler fluxes are discretized using third-order MUSCL scheme [18] in combination with the approximate Riemann solver of Roe [19]. The Non-oscillatory behavior of the MUSCL reconstruction is enforced by applying a slope limiter due to Koren [20].

The system of discrete equations in eqn. 5 is solved iteratively to a pseudo-steady state solution using dual-time stepping $[21,22]$ in the form

$$
\frac{\partial U}{\partial \tau}+R(U)=0,
$$


where, $\tau$ is the dual-time step. In the above equation, $U(\vec{r})$ is written as $U$ for simplicity. Implicit operators are constructed using the diagonalized alternating direction implicit (D-ADI) scheme[23]. The traditional D-ADI scheme only treats the spatial derivative terms implicitly and was found to converge slowly in the presence of time-spectral source terms. Therefore, a sub-iteration type algorithm is employed, where the updates are performed to eqn. 4 as

$$
\frac{U^{k+1}-U^{k}}{\Delta \tau}=D_{t} U^{k}+\hat{R}\left(U^{k+1}\right)+\frac{U^{k}-U^{n}}{\Delta \tau}, \quad k=1,2, . ., s
$$

Here, $n$ is the iteration index and $k$ is the sub-iteration index such that $\left.U^{k}\right|_{k=1}=U^{n}$ and $U^{n+1}=\left.U^{k}\right|_{k=s+1}$. Each of these updates are performed using the traditional D-ADI scheme. Two or three sub-iterations are found to be sufficient to improve the convergence of the simulations presented in this paper. Note that $\Delta \tau$ can vary spatially.

\section{Discrete Adjoint Equations}

For purposes of functional error estimation, a discrete adjoint $[24,25,26,27]$ approach is pursued. In this approach, a numerically exact adjoint is derived from the discretized form of the primal (flow) equations. This is in contrast to the continuous adjoint approach[28, 29,30] in which the adjoint of the primal problem is derived from the continous primal equations and then discretized. It could be argued that the discrete adjoint might be naturally suited for purposes of error estimation because of the exact nature of the solution of the discrete adjoint equations (to machine precision) and also because the discrete formulation is indeed an adjoint of the modified partial differential equation that includes the numerical error. Consider a functional of interest, $f(U, \alpha)$, that is dependent on the flow solution and one or more parameters $\alpha$. The variation of $f, \delta f$ with respect to $\alpha$ is written as

$$
\delta f=\left(\frac{\partial f}{\partial \alpha}+\frac{\partial f}{\partial U} \frac{\partial U}{\partial \alpha}\right) \delta \alpha
$$

This variation can be evaluated if $\frac{\partial U}{\partial \alpha}$ is known, and this can be determined by linearizing the governing equation (eqn. 5) as

$$
\frac{\partial R}{\partial \alpha}+\frac{\partial R}{\partial U} \frac{\partial U}{\partial \alpha}=0
$$

Equations 9 and 10 can be combined as

$$
\delta f=\left(\frac{\partial f}{\partial \alpha}+\frac{\partial f}{\partial U} \frac{\partial U}{\partial \alpha}+\Psi^{T}\left[\frac{\partial R}{\partial \alpha}+\frac{\partial R}{\partial U} \frac{\partial U}{\partial \alpha}\right]\right) \delta \alpha
$$

where $\Psi$ is the adjoint variable introduced as a Lagrangian multiplier. The above equation can be simplified as 


$$
\delta f=\left(\frac{\partial f}{\partial \alpha}+\Psi^{T} \frac{\partial R}{\partial \alpha}\right) \delta \alpha,
$$

if the following equation, called the discrete adjoint equations is satisfied.

$$
\left[\frac{\partial R}{\partial U}\right]^{T} \Psi=-\left[\frac{\partial f}{\partial U}\right]^{T}
$$

Formulation of the Adjoint Problem in Stochastic Space

In the stochastic space $\Omega_{\xi}$, some moment of the functional is considered as the objective function. If, for instance, the mean is required, the following expression

$$
J(U)=\int_{\Omega_{\xi}} f(U) d \xi,
$$

can be used. To compute the above integral, $\Omega_{\xi}$ is divided into $N_{E}$ simplex elements[12, 17], consisting of $N_{S}$ vertices. Deterministic simulations are performed at the $N_{S}$ vertices and the objective function is computed by performing a quadrature on the simplex elements.

$$
J(U)=\sum_{i=1}^{N_{E}} \int_{E_{i}} f(U) d \xi
$$

where, $E_{i}$ represents a division of the random space $\Omega_{\xi}$ into $N_{E}$ simplex elements. To perform this numerical integration, an approximation $\tilde{U}$ is constructed using the solution at the vertices of the simplex elements using a sufficiently high order accurate interpolation. It is evident from this procedure that there is no cross-coupling between the solutions at different sampling points in $\Omega_{\xi}$. Therefore, the solution of the global adjoint problem (as that in section 7.4) reduces to the individual solutions of the adjoint equations at each sampling location.

\section{Adjoint Solution Procedure}

In the current work, a discrete adjoint solver is implemented using the automatic differentiation toolkit Tapenade, developed by Hascoët and Pascual at INRIA [31]. The Tapenade software is written in Java, and it applies source transformation to codes written in Fortran or C. Similar to the flow solution, the adjoint solution is converged iteratively using local pseudo-time stepping and the D-ADI scheme with sub-iterations. 


\section{Error Estimation Procedure}

\subsection{Spatial and Temporal Error Estimation}

Consider a baseline (or coarse) space-time domain $\Omega_{H}$ and a fine space-time domain $\Omega_{h}$ which can be obtained, for instance, by isotropically refining the baseline mesh or by increasing the number of time-spectral instances or by combining both space and time refinement. The goal of this approach is to obtain an accurate estimate of some functional $f\left(U_{h}\right)$ on the fine domain based on the time-spectral flow and adjoint solutions on the coarse domain. To enable this estimation, the flow and adjoint solutions computed on the coarse domain have to be interpolated onto the fine domain (the interpolations are represented by $U_{h}^{H}$ and $I_{h}^{H} \Psi_{H}$, respectively). Expanding the functional and residual about $U_{h}^{H}$ yields,

$$
\begin{aligned}
& f\left(U_{h}\right)=f\left(U_{h}^{H}\right)+\left.\frac{\partial f}{\partial U}\right|_{U_{h}^{H}}\left(U_{h}-U_{h}^{H}\right), \\
& R\left(U_{h}\right)=R\left(U_{h}^{H}\right)+\left.\frac{\partial R}{\partial U}\right|_{U_{h}^{H}}\left(U_{h}-U_{h}^{H}\right) .
\end{aligned}
$$

If a discrete numerical scheme can target (to machine precision) $R\left(U_{h}\right)=0$, one can write

$$
\begin{aligned}
f\left(U_{h}\right) & =f\left(U_{h}^{H}\right)+\left.\frac{\partial f}{\partial U}\right|_{U_{h}^{H}}\left(U_{h}-U_{h}^{H}\right)+\left(I_{h}^{H} \Psi_{H}\right)^{T}\left[R\left(U_{h}\right)-R\left(U_{h}^{H}\right)+R\left(U_{h}^{H}\right)\right] \\
& =f\left(U_{h}^{H}\right)+\left[\left.\frac{\partial f}{\partial U}\right|_{U_{h}^{H}}+\left.\left(I_{h}^{H} \Psi_{H}\right)^{T} \frac{\partial R}{\partial U}\right|_{U_{h}^{H}}\right]\left(U_{h}-U_{h}^{H}\right)+\left(I_{h}^{H} \Psi_{H}\right)^{T} R\left(U_{h}^{H}\right) .
\end{aligned}
$$

Now, define the transpose of adjoint residual operator as

$$
R^{\Psi}(\cdot)=\left.\frac{\partial f}{\partial U}\right|_{U_{h}^{H}}+\left.(\cdot)^{T} \frac{\partial R}{\partial U}\right|_{U_{h}^{H}} .
$$

This leads to the expression derived by Venditti and Darmofal [5] for an estimate of the numerical discretization error:

$$
\begin{aligned}
f\left(U_{h}\right) & =f\left(U_{h}^{H}\right)+\left(I_{h}^{H} \Psi_{H}\right)^{T} R\left(U_{h}^{H}\right)+R^{\Psi}\left(I_{h}^{H} \Psi_{H}\right)\left(U_{h}-U_{h}^{H}\right) \\
& =f\left(U_{h}^{H}\right)+\varepsilon_{c c}+\varepsilon_{r e} .
\end{aligned}
$$


In the above equation, the first two terms can be evaluated by post-processing the coarse domain flow and adjoint solutions, while the third term is not computable. In this work, the high order spatial interpolation operator is taken to be a third order accurate MUSCL reconstruction[20]. The temporal interpolation is performed using Fourier interpolants. Note that, for the error estimation to be accurate, it would be desirable for $\left|\varepsilon_{r e}\right|$, the remaining error term, to be small relative to $\left|\varepsilon_{c c}\right|$, the computable error. A possible way of achieving this could be through mesh adaptation and temporal refinement.

\subsection{Stochastic Error Estimation}

In computing the statistical average [32], the integral in eqn. 15 is approximated using a quadrature on simplex elements which is exact for polynomials of degree $s$,

$$
\left.J \approx \sum_{i=1}^{N_{E}} \sum_{j=1}^{N_{q}} w_{i j} f\left(U_{i j}\right)\right)+O\left(\Delta \xi^{s+1}\right)
$$

Here $N_{E}$ is the number of simplex elements and $N_{q}$ is the number of quadrature points. For the purpose of clarity, the subscripts $(.)_{i j}$ will be used to denote the value of the quantity (.) at the $j^{\text {th }}$ Gaussian quadrature point in element $i$. The quantities $f, U$ and $R$ can depend explicitly on the stochastic variables without changing the formulation, but the dependence is not indicated for the purpose of clarity.

Note that $U$ is known only at the vertices of the simplices, $\xi_{k}, k=1, \ldots, N_{s}$. Using these vertex values of $U$, the solution can be reconstructed at the Gaussian points using either a linear, quadratic or higher order reconstruction technique. If the reconstructed flow and adjoint solutions are $\tilde{U}$ and $\tilde{\Psi}$, respectively, and assuming linearity,

$$
f\left(U_{i j}\right)=f\left(\tilde{U}_{i j}\right)+\frac{\partial f}{\partial U}\left(\tilde{U}_{i j}\right)\left(U_{i j}-\tilde{U}_{i j}\right) .
$$

Since $R\left(U_{i j}\right)=0$,

$$
\begin{aligned}
f\left(U_{i j}\right) & =f\left(\tilde{U}_{i j}\right)+\frac{\partial f}{\partial U}\left(\tilde{U}_{i j}\right)\left(U_{i j}-\tilde{U}_{i j}\right)+\tilde{\Psi}_{i j}^{T}\left[R\left(U_{i j}\right)-R\left(\tilde{U}_{i j}\right)+R\left(\tilde{U}_{i j}\right)\right] \\
& =f\left(\tilde{U}_{i j}\right)+\tilde{\Psi}_{i j}^{T} R\left(\tilde{U}_{i j}\right)+\left[\frac{\partial f}{\partial U}\left(\tilde{U}_{i j}\right)+\tilde{\Psi}_{i j}^{T} \frac{\partial R}{\partial U}\left(\tilde{U}_{i j}\right)\right]\left(U_{i j}-\tilde{U}_{i j}\right) \\
& =f\left(\tilde{U}_{i j}\right)+\varepsilon_{c c}+\varepsilon_{r e} .
\end{aligned}
$$

Note that the procedure mirrors the spatial error estimation procedure of Venditti and Darmofal [5]. The first two terms in the above equation are computable, while the third term is not. If the remaining error is small, the mean value of the functional is given by 


$$
\left.J \approx \sum_{i=1}^{N_{E}} \sum_{j=1}^{N_{q}} w_{i j}\left\{f\left(\tilde{U}_{i j}\right)\right)+\tilde{\Psi}_{i j}^{T} R\left(\tilde{U}_{i j}\right)\right\}
$$

The elements used in the stochastic space can be linear (P1), quadratic (P2) or higher order simplex elements. In the current work, only P1 and P2 elements are used. Figure 1 shows a schematic of P1 and P2 elements used in 1D and 2D stochastic space. The sample points are represented as solid circles. To integrate the functional within each element, a four point quadrature rule is used in 1D space. In 2D stochastic space, the integration is performed using the symmetric Dunavant quadrature rule [33]. The quadrature points are located inside the simplex elements; for P1 elements, four quadrature points are sufficient while P2 elements require seven quadrature points. These choices ensure that quadrature errors are lower than interpolation errors.

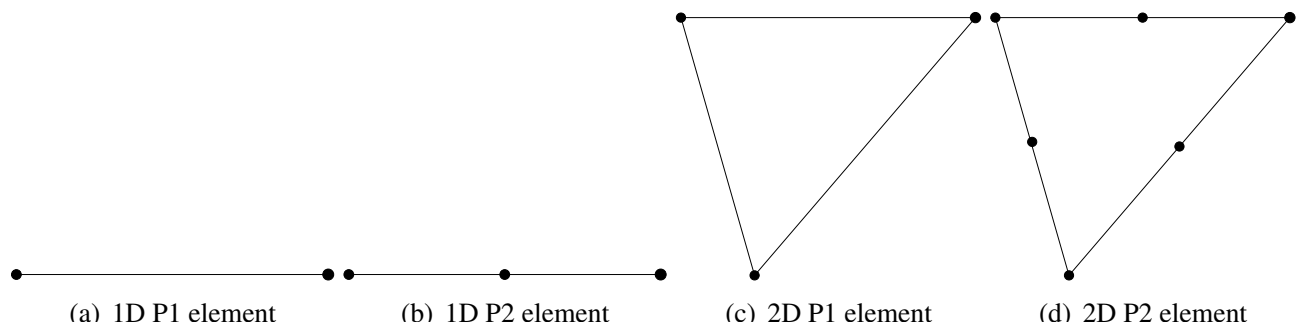

(a) 1D P1 element

(b) $1 \mathrm{D}$ P2 element

(c) $2 \mathrm{D}$ P1 element

(d) 2D P2 element

Figure 1: Schematic of elements in 1D and 2D stochastic space. Sample points are marked by solid circle symbols.

\section{Flow Solver Verification and Validation}

The primary validation test case chosen for this work is based on an experimental setup of Oler et al. [34]. The setup consists of a one-bladed vertical axis wind turbine operating in a water tank. The blade uses a NACA 0015 airfoil and has a chord length of $15.24 \mathrm{~cm}$. The rotor diameter is $122 \mathrm{~cm}$, resulting in a chord to radius ratio $(c / R)$ of 0.25 . The rotor operates at a tip speed of $45.7 \mathrm{~cm} / \mathrm{s}$, yielding a chord-based Reynolds number of 67000 . Force measurements are available at three different tip to wind-speed ratio (TSR) of 2.5, 5.0 and 7.5. A schematic of the setup is shown in Fig. 2(a).

Simulations are performed at a TSR of 7.5 in two dimensions. To avoid convergence issues associated with low Mach number simulations using the compressible solver, the rotational Mach number is set to 0.225 . Therefore, the freestream Mach number, referred to as normalized wind speed, is 0.03 . The baseline grid (will be referred to throughout the paper) used for the simulation is a C-type grid with $225 \times 65$ points and is shown in Fig. 2(b).

Figure 3 shows the comparison of the predicted normal $\left(C_{N}\right)$ and tangential $\left(C_{T}\right)$ force coefficients as a function of azimuth $(\xi)$ with the experimental data. Shown are the results from inviscid and RANS simulations (using the Spalart-Allmaras [35] turbulence model). The normal force is predicted to within $10 \%$ of the experimental value 


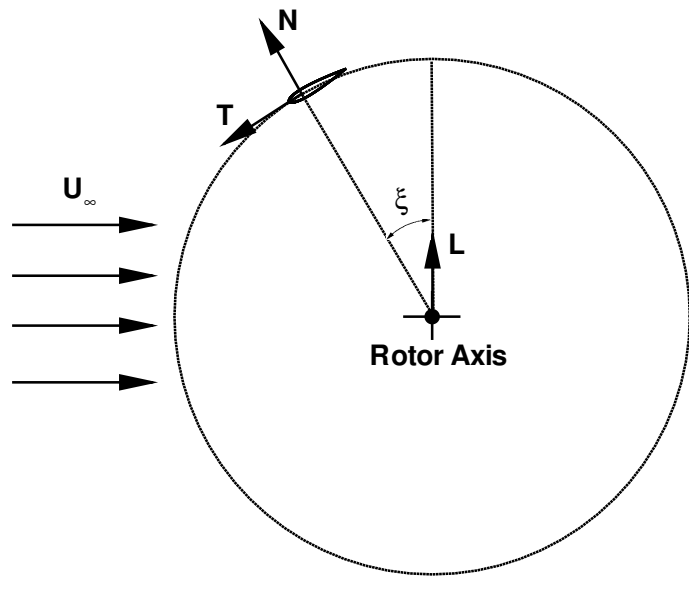

(a) Flow schematic

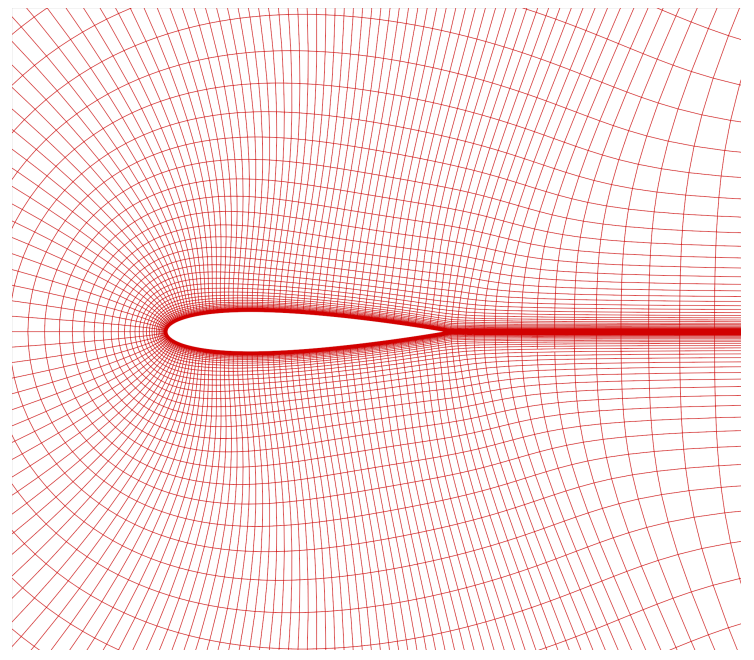

(b) Baseline grid $(225 \times 65)$

Figure 2: Flow schematic and computational mesh for vertical axis wind-turbine calculation.

in both viscous and inviscid calculations. One of the main reasons for the discrepancy is the assumption of two dimensionality in the simulation whereas the experiment used a short aspect ratio blade. Other geometric features such as the hub of the turbine and the connecting rod have also been ignored in the present simulations. As expected, the tangential force prediction has higher discrepancy using the inviscid calculation. For the purpose of verifying the error estimation procedure, the inviscid setup should nevertheless serve as a good test case as it contains the relevant flow phenomena.

In Fig. 4, the results from the time spectral simulations using different number of time instances are compared against the time marching result. The time marching solution is obtained using 2880 time-steps per revolution and the result after 20 revolutions is plotted. The time spectral solution is seen to converge to the time marching result when the number of time instances is increased, thus verifying the implementation in the flow solver. Fig. 5 shows the power coefficient $\left(C_{P}\right)$ as a function of azimuth on the baseline grid as well as on a fine grid obtained by refining the mesh in both directions. The instantaneous power is taken to be the product of instantaneous tangential force and the rotational velocity. The time-marching results are seen to be fairly grid-converged. It is important to note that on the fine grid, a larger number of time-spectral instances is required for accurate numerical resolution.

\section{Verification of the Adjoint Solver}

Following the validation of the flow solver, the adjoint solver is verified by comparing the adjoint-computed sensitivities to those obtained using finite difference. The first problem considered is an inviscid steady flow past a NACA 0015 airfoil at $10^{\circ}$ angle of attack and Mach 0.15 . The sensitivities of lift coefficient $\left(C_{l}\right)$ with respect to 


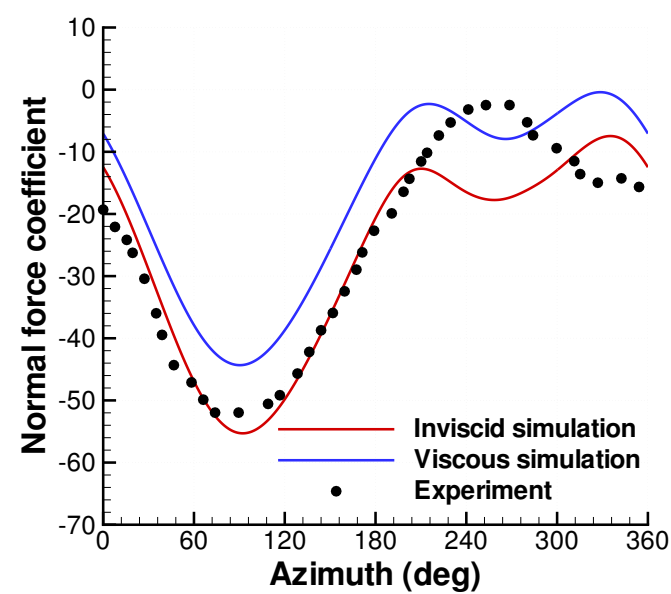

(a) Normal force coefficient

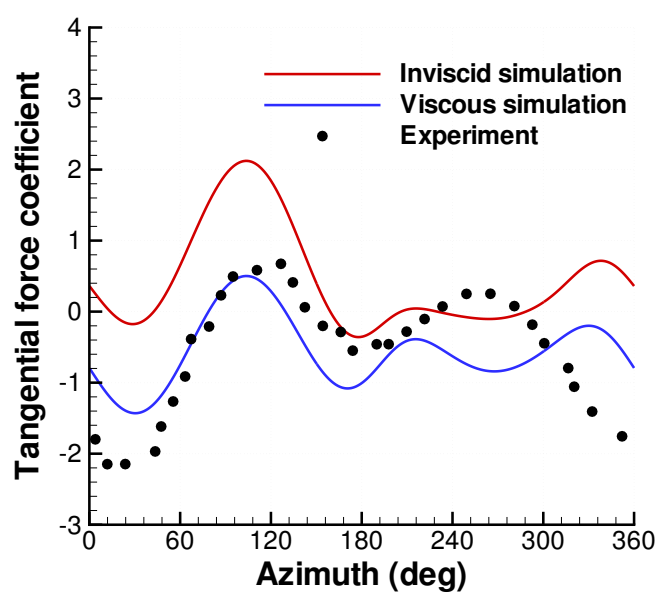

(b) Tangential force coefficient

Figure 3: Comparison of force time histories between CFD and experiment for Oler problem.

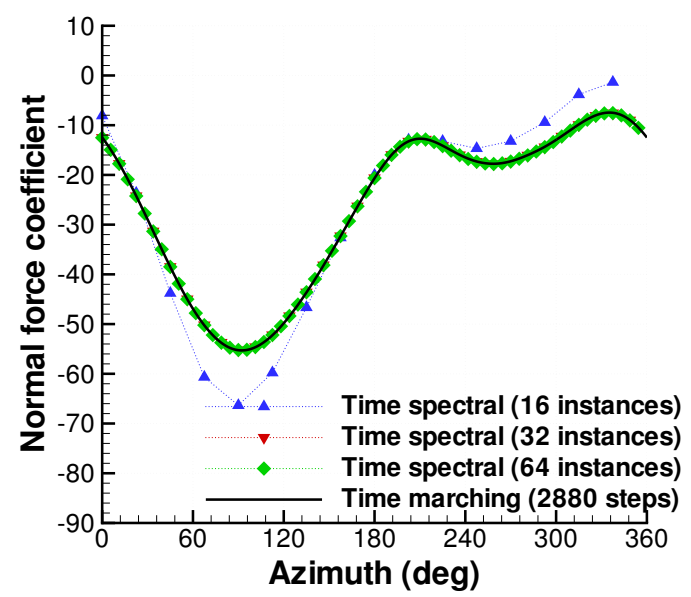

(a) Normal force coefficient

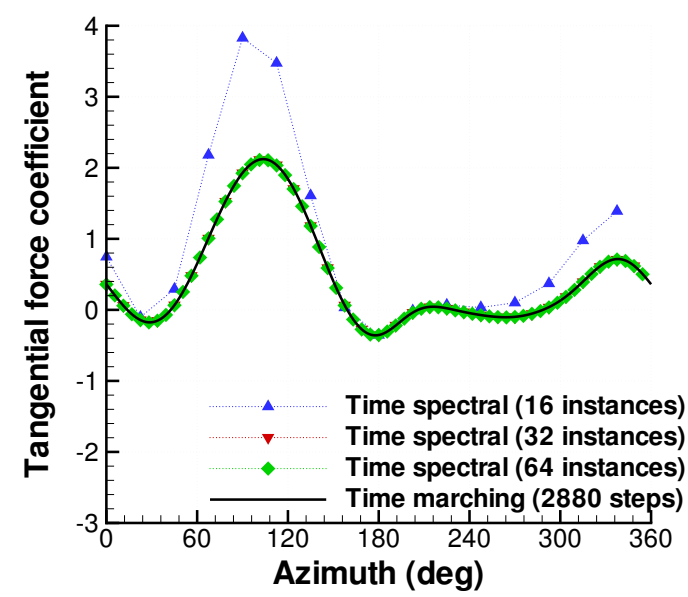

(b) Tangential force coefficient

Figure 4: Comparison of force time histories for different time spectral instances for inviscid Oler problem.

freestream Mach number and angle of attack, shown in Table 1, are highly accurate. Contour plots of density and adjoint density are shown in Fig. 6 for reference.

Table 2 compares the sensitivities of vertical force and power coefficients (both averaged over one period) with respect to freestream Mach number for the inviscid Oler VAWT problem described in the previous section. The results are computed using 32 time-spectral instances. The finite difference results are obtained with a step size of 0.00001 . The sensitivities are again confirmed to be highly accurate, thus verifying the time-spectral adjoints. Figure 7 shows the contour of y-velocity and adjoint y-velocity at one azimuth location. 


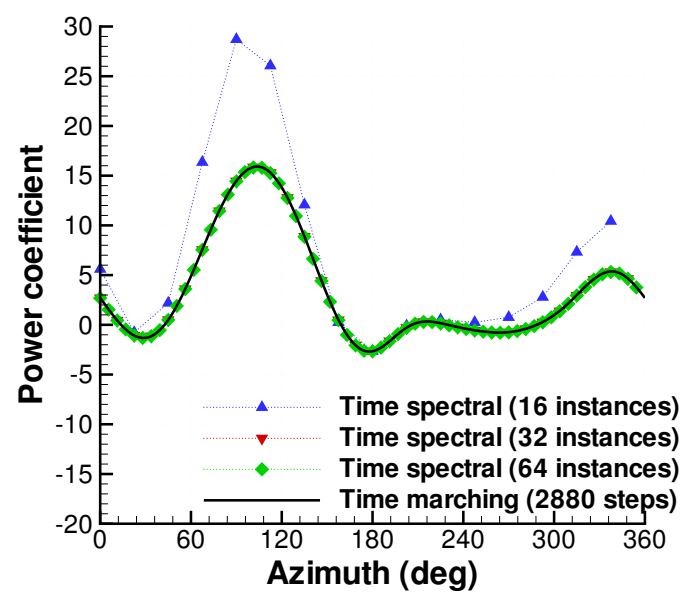

(a) Baseline grid $(225 \times 65)$

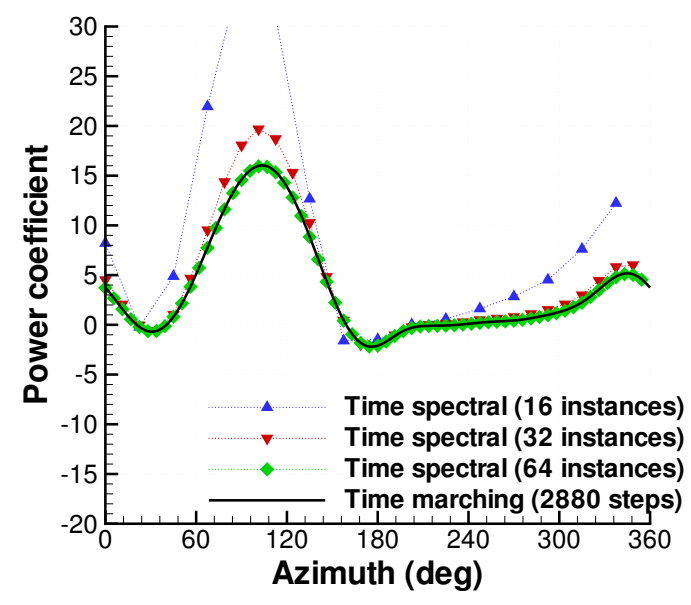

(b) Fine grid $(449 \times 129)$

Figure 5: Comparison of power coefficient time histories for baseline and fine grid for inviscid Oler problem.

\begin{tabular}{|c|c|c|c|c|}
\hline \multirow{2}{*}{$C_{l}$} & \multicolumn{2}{|c|}{$d C_{l} / d M_{\infty}$} & \multicolumn{2}{c|}{$d C_{l} / d \alpha$} \\
\cline { 2 - 5 } & Adjoints & FD & Adjoints & FD \\
\hline 1.23652 & 0.20734 & 0.20734 & 0.12131 & 0.12131 \\
\hline
\end{tabular}

Table 1: Comparison of sensitivities of lift coefficient $\left(C_{l}\right)$ using adjoints and finite difference for steady NACA 0015 problem.

\begin{tabular}{|c|c|c|c|c|c|}
\hline \multirow{2}{*}{$C_{L}$} & \multicolumn{2}{|c|}{$d C_{L} / d M_{\infty}$} & \multirow{2}{*}{$C_{P}$} & \multicolumn{2}{c|}{$d C_{P} / d M_{\infty}$} \\
\cline { 2 - 3 } \cline { 5 - 6 } & Adjoints & Finite difference & & Adjoints & Finite difference \\
\hline 1.50894 & 22.94781 & 22.94781 & 3.31702 & 216.59346 & 216.59346 \\
\hline
\end{tabular}

Table 2: Comparison of sensitivities of average vertical force coefficient $\left(C_{L}\right)$ and average power coefficient $\left(C_{P}\right)$ using adjoints and finite difference for inviscid Oler problem.

\section{Error Estimation Results}

\subsection{Spatial Error Estimation}

The accuracy of the spatial error estimation approach is verified using the steady (NACA 0015) and unsteady (Oler) test cases introduced in the previous section. For the NACA 0015 problem, the functional is chosen to be the lift coefficient and the error estimation is performed on five levels of grid refinement. The baseline grid is the third in the series and is the same as the one used for the Oler problem. Both the flow and adjoint solutions are reconstructed from a coarse mesh onto the next level fine mesh using a finite-element based bi-cubic interpolation. Gradient limiters were not used as the solution is smooth in the entire domain. The error estimation results are shown in Fig. 8. Figure 8(a) shows the functional $(f)$ as computed on any grid and the adjoint-corrected functional $\left(f+\varepsilon_{c c}=f\left(U_{h}^{H}\right)+\left(I_{h}^{H} \Psi_{H}\right)^{T} R\left(U_{h}^{H}\right)\right)$ that estimates the functional on the grid at the next level of refinement. It is apparent from this figure that the error estimation approximates the fine grid functional to a good degree. Figure 8(b) 


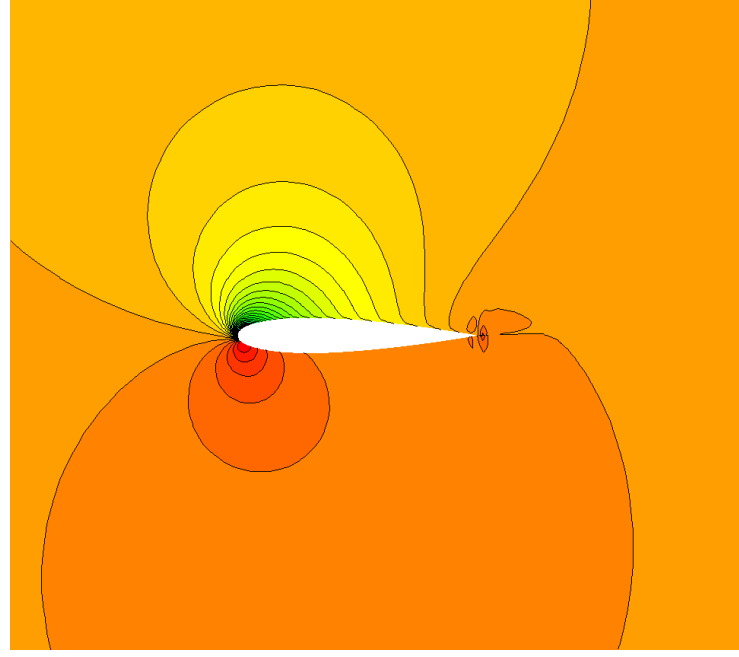

(a) Density contours

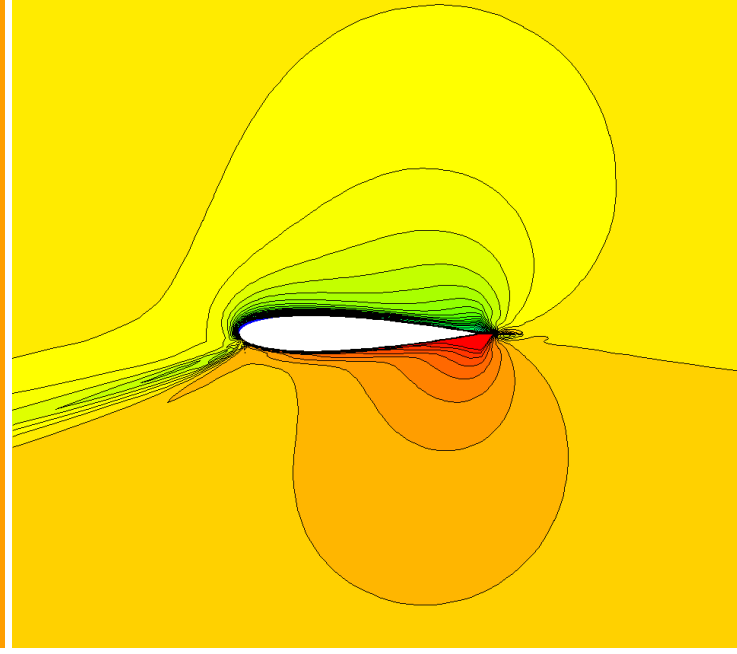

(b) Adjoint density contours

Figure 6: Flow and adjoint contours for steady NACA 0015 problem.

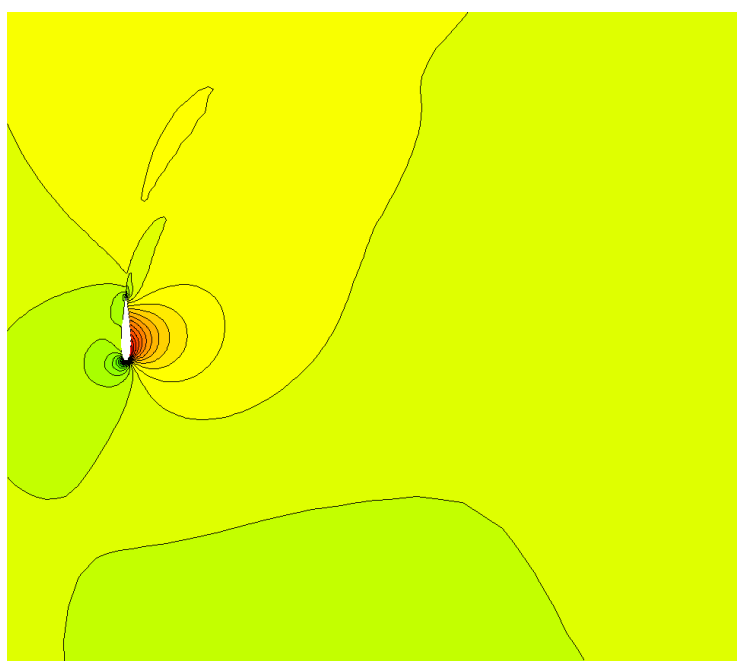

(a) y-velocity contours

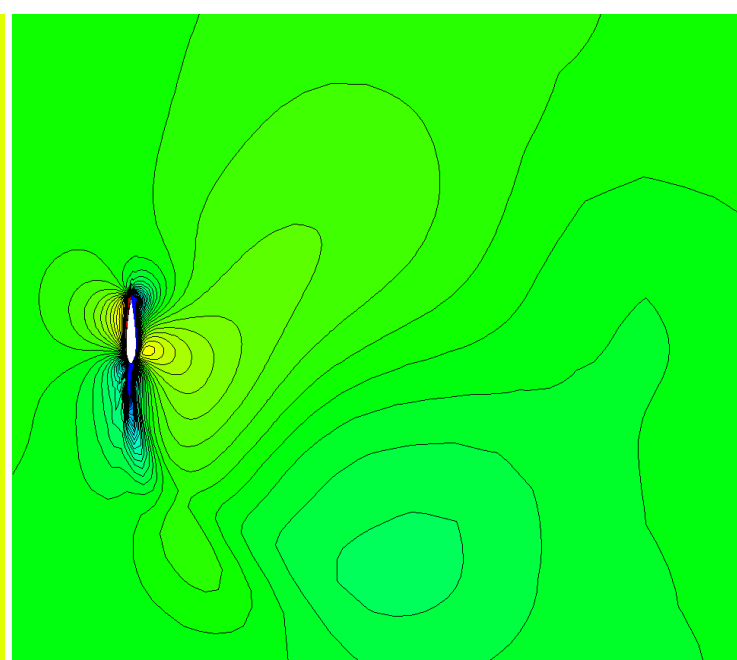

(b) Adjoint y-velocity contours

Figure 7: Flow and adjoint contours for inviscid Oler problem.

compares the estimated error with the relative error, defined as $\left|f\left(U_{h}\right)-f\left(U_{h}^{H}\right)\right|$, and the remaining error. Figure 8(c) plots the ratio of estimated error to the relative error. From this exercise, it is confirmed that the error estimation procedure is able to recover most of the spatial discretization error and provides a super-convergent estimate of the functional.

A similar error estimation study is performed for the inviscid Oler problem. Four grid levels are used, two of the levels are coarser than the baseline grid and one of them is finer. The solution is obtained using 64 time-spectral instances. The functional is chosen to be the average power coefficient. Both the flow and adjoint solutions are again 


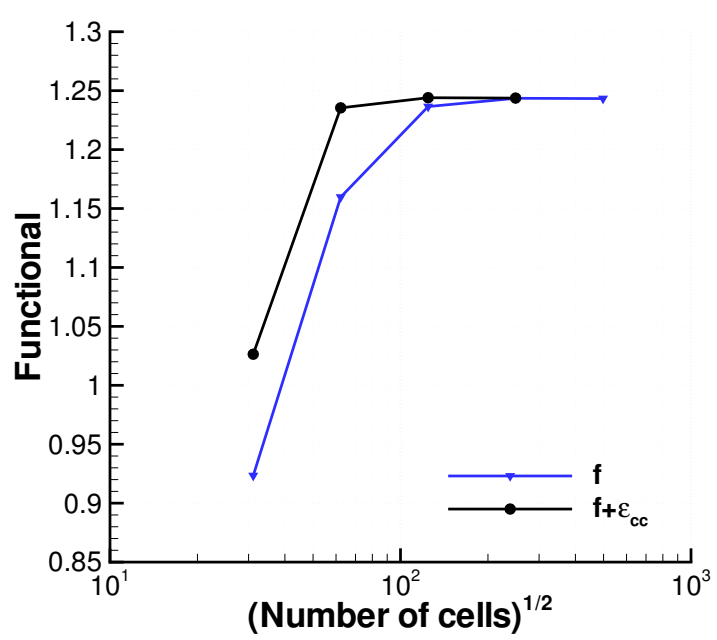

(a) Functional

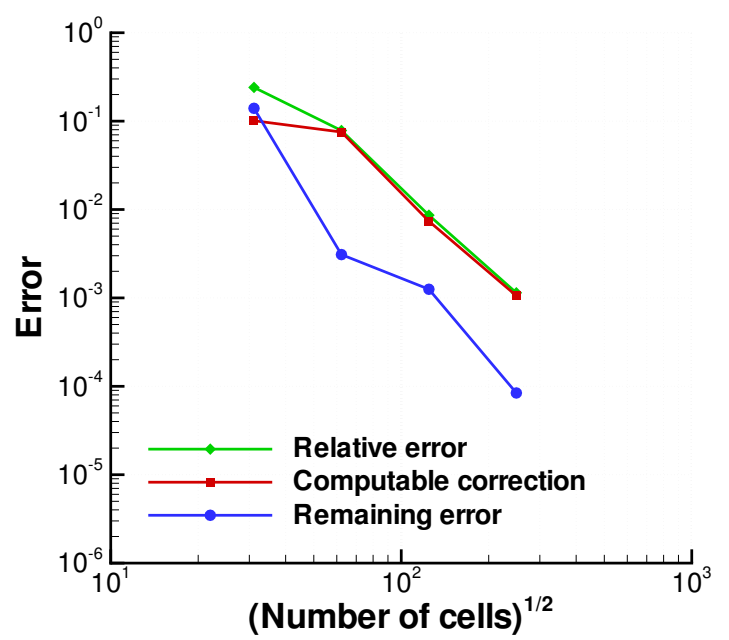

(b) Errors

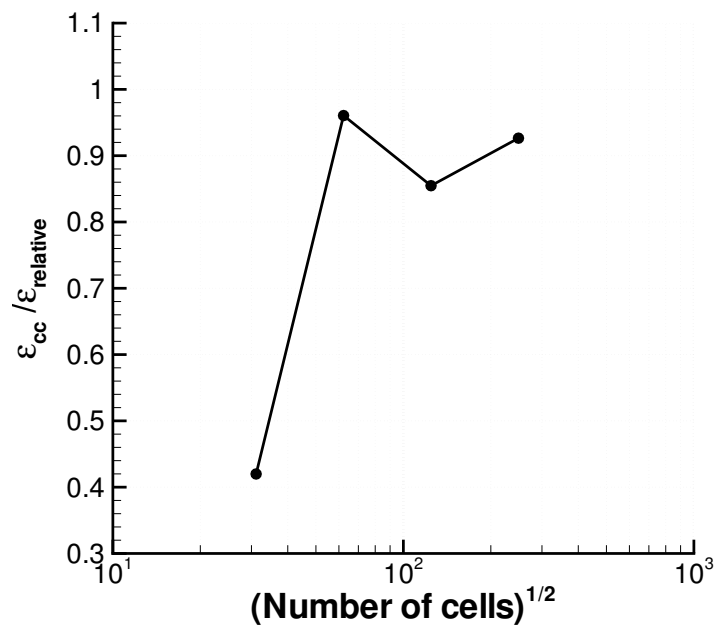

(c) $\varepsilon_{c c} / \varepsilon_{\text {relative }}$

Figure 8: Results from spatial error estimation for NACA 0015 problem.

interpolated from a coarse mesh onto the fine mesh using bi-cubic interpolation. The results are shown in Table 3. A good recovery of spatial error and an accurate estimation of the functional is observed for this problem as well.

\begin{tabular}{|c|c|c|c|}
\hline Mesh & $f$ & $f+\varepsilon_{c c}$ & $\varepsilon_{c c} / \varepsilon_{\text {relative }}$ \\
\hline V. Coarse Grid $(57 \times 17)$ & -6.9474 & 1.3781 & 0.97 \\
Coarse Grid $(113 \times 33)$ & 1.6552 & 3.6199 & 1.17 \\
Baseline Grid $(225 \times 65)$ & 3.3145 & 3.6386 & 1.18 \\
Fine Grid $(449 \times 129)$ & 3.5813 & - & - \\
\hline
\end{tabular}

Table 3: Spatial error estimation results for inviscid Oler problem. 


\subsection{Temporal Error Estimation}

The temporal error estimation procedure is verified for the inviscid Oler problem. The error estimation is performed on five different successively refined time intervals as shown in Table 4. The computations are performed on the baseline spatial grid. Both the flow and adjoint solutions are spectrally reconstructed when interpolating from a coarser to a finer level of the time-spectral domain. The results, tabulated in Table 4, show that the error estimation provides the correct sign of the error for all time domains. Almost all the error is recovered for the finest time domain, but only small proportions of the actual relative error are recovered on the coarser time domains. The reason for poor error recovery for the coarse time domains is investigated by computing the remaining error. The remaining error can be easily computed if the flow solution on the finer time domain is available. The estimation of remaining error, shown in Table 5, clearly indicates that a huge fraction of the total error is not computatable on the coarser time domain. This also suggests that a different interpolation strategy for the adjoint solution might be required to obtain more accurate estimates

\begin{tabular}{|c|c|c|c|}
\hline Time instances & $f$ & $f+\varepsilon_{c c}$ & $\boldsymbol{\varepsilon}_{c c} / \boldsymbol{\varepsilon}_{\text {relative }}$ \\
\hline 16 & 6.8604 & 6.7832 & 0.025 \\
24 & 3.7661 & 3.7633 & 0.006 \\
32 & 3.3170 & 3.3167 & 0.103 \\
48 & 3.3145 & 3.3145 & 0.989 \\
64 & 3.3145 & - & - \\
\hline
\end{tabular}

Table 4: Temporal error estimation results for inviscid Oler problem.

\begin{tabular}{|c|c|}
\hline Time instances & $\varepsilon_{r e} / \varepsilon_{\text {relative }}$ \\
\hline 16 & 1.020 \\
24 & 1.012 \\
32 & 0.853 \\
48 & 0.010 \\
64 & - \\
\hline
\end{tabular}

Table 5: Estimation of remaining temporal error for inviscid Oler problem.

The strategy that we are proposing to improve the temporal reconstruction of the adjoint solution is to converge the adjoint residual on the refined time domain (the domain onto which the coarser solution is interpolated) for a few orders of magnitude starting from the spectrally interpolated adjoint solution about the spectrally interpolated flow solution. The adjoint solution obtained in this fashion will be referred to as smoothed adjoint solution in the remainder of the paper. Table 6 shows the estimated error for the Oler problem using the smoothed adjoint solution obtained by converging the adjoint residual by two orders of magnitude. The new estimates clearly provide excellent recovery of the time error even on the coarser domains. For these problems, converging the adjoint residual by two orders of magnitude requires less than $1 \%$ of the computational cost required to obtain the flow and adjoint solution on the finer 
domain, thus making this reconstruction strategy practically feasible.

\begin{tabular}{|c|c|}
\hline Time instances & $\varepsilon_{c c} / \varepsilon_{\text {relative }}$ \\
\hline 16 & 1.042 \\
24 & 1.013 \\
32 & 0.959 \\
48 & 1.001 \\
64 & - \\
\hline
\end{tabular}

Table 6: Temporal error estimation results for inviscid Oler problem using new reconstruction strategy.

\subsection{Combined Spatial and Temporal Error Estimation}

In this section, the error estimation procedure is evaluated by performing a combined refinement in space and time. Earlier it was observed that the Oler problem simulated on a finer grid requires a larger number of time-spectral instances to converge to the time-marched solution. Therefore, a good recovery of the spatio-temporal error can provide significant savings to the computational cost of these simulations.

The domain referred to as "baseline" in this section uses the baseline grid and is simulated with 32 time-spectral instances. A finer grid, obtained by refining both spatial directions, is simulated with twice the number of time instances. The error estimation results for the average power coefficient is shown in Table 7. The results clearly show good functional recovery, thus exemplifying the advantages of using an error estimation procedure. The error recovery can be seen to be comparable to the spatial error estimation results, clearly indicating that the spatial component of error is dominant for this problem. Note that using smoothed adjoint solutions did not have a significant effect on the error estimates.

\subsection{Stochastic Error Estimation}

The verification of the stochastic error estimation procedure is now pursued on the inviscid Oler problem in both 1D and 2D stochastic space. The 1D problem involves a situation where the normalized wind speed varies (in a uniform fashion) between 0.025 and 0.035 . For the two-parameter problem, in addition to the aforementioned wind speed variation, the attachment position of the blade with the spoke of the hub varies uniformly between 0.2 and 0.3 chords. The objective functional is taken to be the mean power coefficient over the parameter range. The simulations

\begin{tabular}{|c|c|c|c|}
\hline Domain & $f$ & $f+\varepsilon_{c c}$ & $\varepsilon_{c c} / \varepsilon_{\text {relative }}$ \\
\hline V. Coarse $(57 \times 17 \times\{8\})$ & -6.4309 & 2.8003 & 1.12 \\
Coarse $(113 \times 33 \times\{16\})$ & 1.8197 & 4.2433 & 1.56 \\
Baseline $(225 \times 65 \times\{32\})$ & 3.3170 & 3.6583 & 1.25 \\
Fine $(449 \times 129 \times\{64\})$ & 3.5813 & - & - \\
\hline
\end{tabular}

Table 7: Combined spatial and temporal error estimation results for inviscid Oler problem. $\{$.$\} represent time instances.$ 
are performed on the baseline grid with 32 time-spectral instances. Quadratic (P2) elements are used in the stochastic space. An adaptive sampling procedure described later in section 8 , is used to sample the stochastic points. However, since the objective functional has a smooth variation over the parameter space, the final sample point distribution is fairly uniform in both 1D and 2D problems as seen in Fig. 9. The results of the stochastic error estimation is shown in Figs. 10 and 11. The effectiveness of adding the adjoint-based computable correction is confirmed. The adjointcorrected estimate of the statistical moment reaches a converged value value at a rapid rate and the adjoint corrections recover a large percentage of the error.

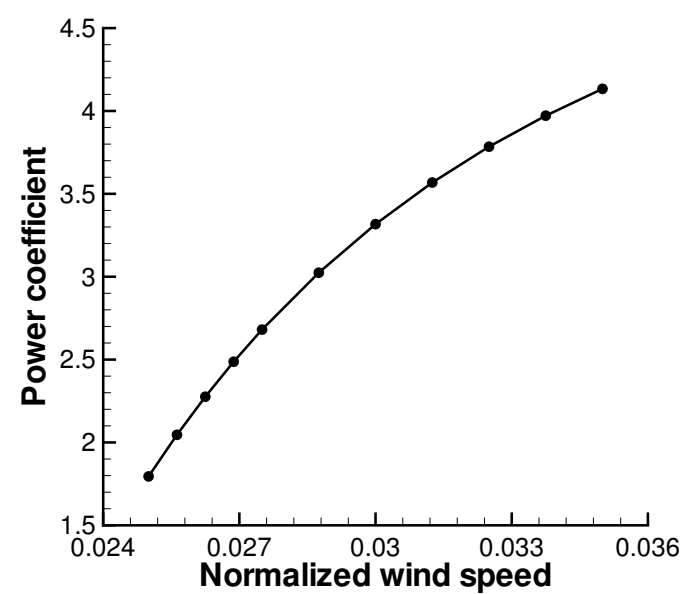

(a) $1 \mathrm{D}$

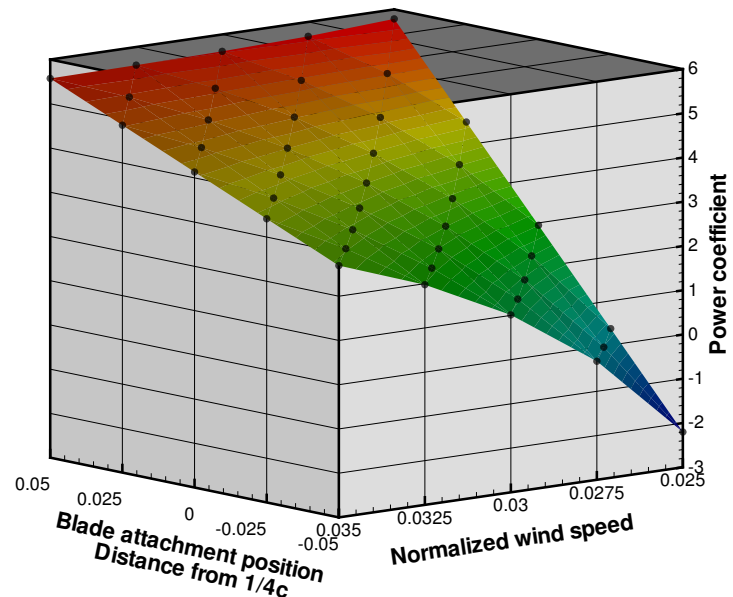

(b) $2 \mathrm{D}$

Figure 9: Final response surfaces in 1D and 2D stochastic space for Oler VAWT problem.

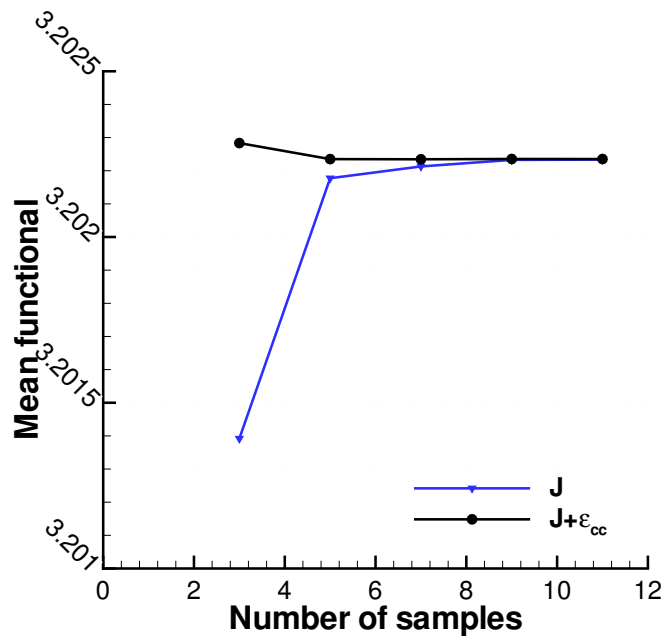

(a) Functional

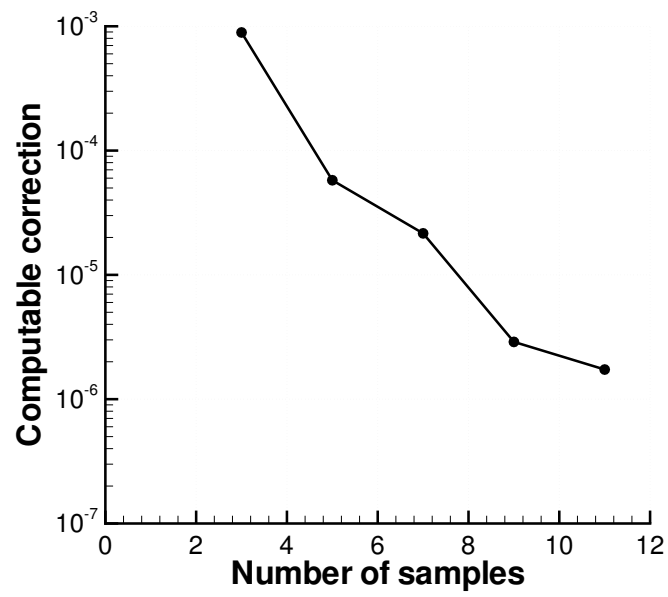

(b) Error

Figure 10: 1D stochastic error estimation results for the Oler VAWT problem. 


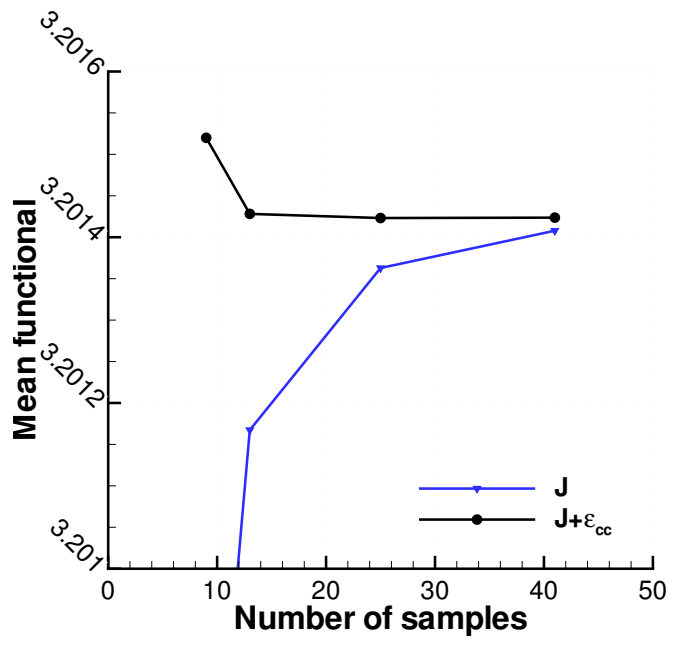

(a) Functional

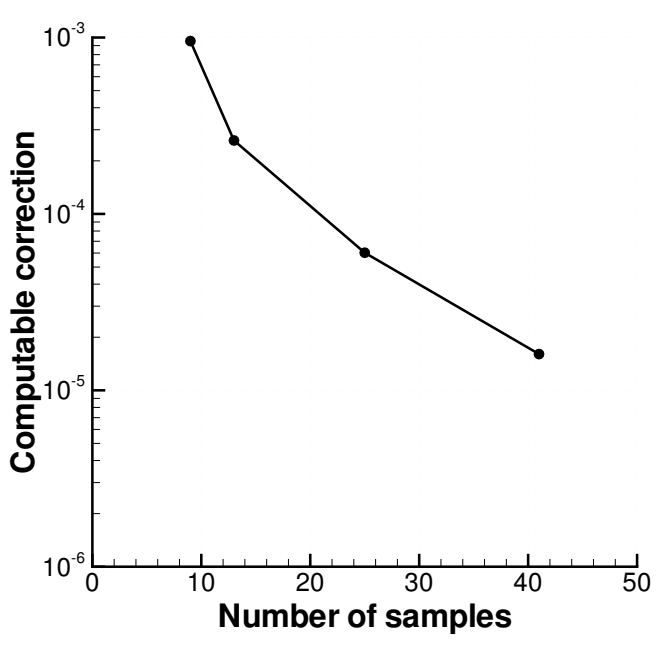

(b) Error

Figure 11: 2D stochastic error estimation results for inviscid Oler problem.

\subsection{Budget of Spatial, Temporal and Stochastic Errors}

Figure 12(a) shows the contour of the magnitude of the estimated stochastic sampling error in the parametric space obtained on the final stochastic mesh. The errors are seen to be uniformly small in the entire domain. Figures 12(b) and 12(c), respectively show the contours of spatial and temporal error magnitude in the stochastic space. The errors shown are the respective computable corrections calculated at all the stochastic sample points by interpolating the flow and adjoint solution onto either a doubly refined grid or onto a domain with twice the number of time instances. The temporal errors are obtained using the smoothed adjoint solution obtained by converging the adjoint residual for two orders of magnitude. The spatial and temporal errors are seen to be higher at lower wind speeds ostensibly because of the more complex near-wake system resulting from the lower tip speed ratios.

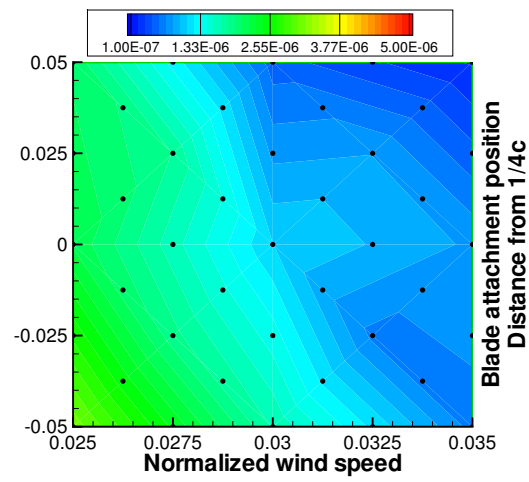

(a) Stochastic error

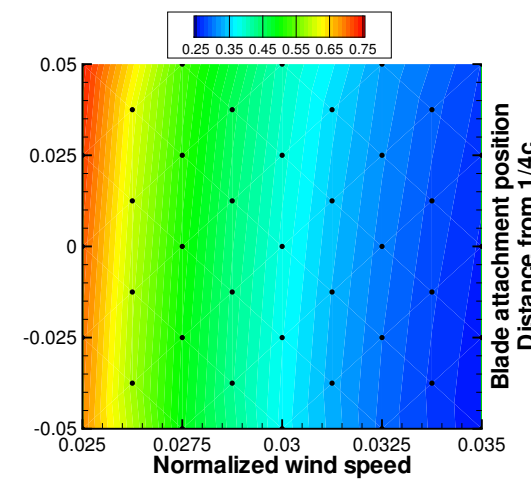

(b) Spatial error

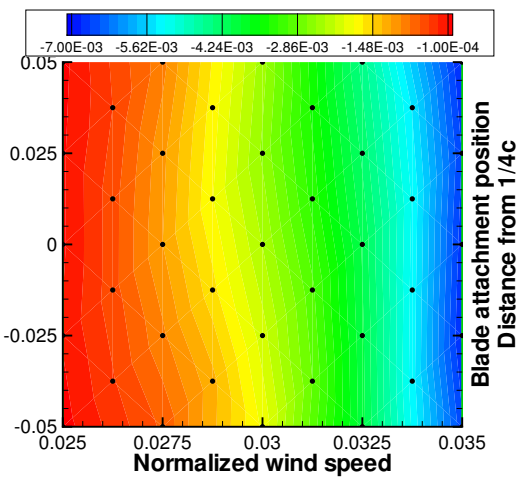

(c) Temporal error

Figure 12: Contours of different errors (computable correction) in stochastic space for inviscid Oler problem. 
To balance various errors, computations are performed on a doubly refined grid with a smaller number of sampling points in the same stochastic space. To account for the fact that solution on a finer grid requires a larger number of time-spectral instances to converge to the time-marching solution, the number of time-spectral instances is doubled to 64. Figure 13 shows various error contours on the new domain. Clearly, the gap between the spatial and the stochastic errors has decreased, but the spatial errors still dominate the problem. Quite clearly, in the present situation, the most appropriate strategy would be to further improve the spatial resolution of samples.

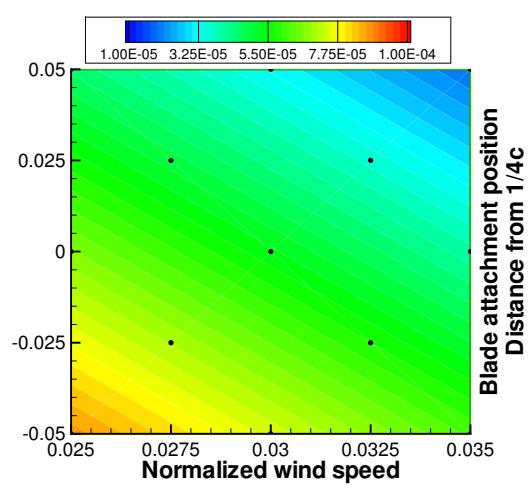

(a) Stochastic error

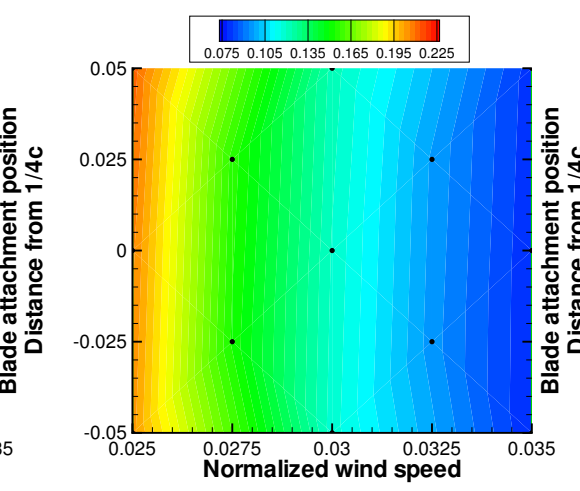

(b) Spatial error

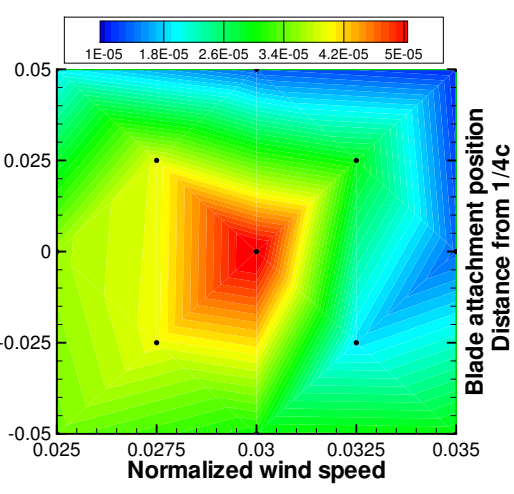

(c) Temporal error

Figure 13: Contours of different errors (computable correction) in stochastic space for inviscid Oler problem computed on refined spatial grid.

\section{Stochastic Sampling and Adaptation Procedure}

The adjoint based error estimate of the functional can be used to adaptively sample the solution in the stochastic space leading to a goal-oriented adaptive approach to uncertainty quantification. The remaining error, which is not precisely computable, can be used as an indicator to decide where to perform additional sampling. Since we do not know the exact solutions $U_{i j}$ and $\Psi_{i j}$ we need to estimate the terms $\left(U_{i j}-\tilde{U}_{i j}\right)$ and $\left(\Psi_{i j}-\tilde{\Psi}_{i j}\right)$ [refer to section 4.2 for the definition of these terms]. This can be achieved by using two reconstruction operators of different accuracy [12]. Let $L$ and $H$ denote lower and higher order reconstruction operators in stochastic space, then an estimate of remaining error in stochastic element $i$ is given by

$$
\varepsilon_{i}=\left|\sum_{j=1}^{N_{q}} w_{i j}\left\{\left[(H \Psi)_{i j}-(L \Psi)_{i j}\right]^{T} R\left(\tilde{U}_{i j}\right)+R^{\Psi}\left(\tilde{\Psi}_{i j}\right)\left[(H U)_{i j}-(L U)_{i j}\right]\right\}\right|
$$

where, $R^{\Psi}(\cdot)$ is the transpose of adjoint residual operator given by $\frac{\partial f}{\partial U}\left(\tilde{U}_{i j}\right)+(\cdot)^{T} \frac{\partial R}{\partial U}\left(\tilde{U}_{i j}\right)$. In the present work, the operator $H$ is chosen to be the same as that used to calculate $\tilde{U}_{i j}$ and $\tilde{\Psi}_{i j}$, and the operator $L$ is chosen to be one order lower. For example, when using linear reconstruction in stochastic space, $H$ and $L$ are chosen as linear and constant 
in element, respectively. The element error, $\varepsilon_{i}$ is then used as an adaptation indicator. If an element has a large local error above a set tolerance level, then it can be divided into two or more elements by adding new sample points. In the current work, the elements in both 1D and 2D stochastic space is divided into two when flagged for adaptation. The 1D element is divided by splitting the element at the mid-point, while a 2D triangular element is divided by joining the mid-point of the largest edge with the vertex opposite to it. A schematic of initial 2D elements formed by joining the four boundary stochastic point and a sketch of a 2D element getting split into two is shown in Figs. 14(a) and 14(b), respectively. The total remaining error obtained by summing the errors in all the elements and the total number of sample points is used as a stopping criterion for the adaptation.

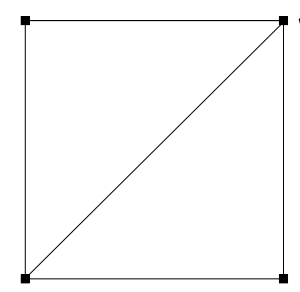

(a) Initial elements

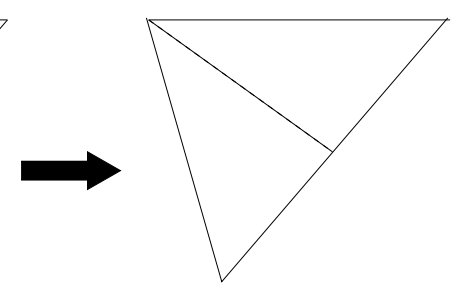

(b) Element split into two when flagged

Figure 14: Schematic of elements in 2D stochastic space. Solid square symbols are boundary samples of the stochastic space.

To demonstrate the effectiveness of adaptive stochastic sampling, the variation of the two parameters (normalized wind speed and blade attachment position with the spoke of the hub) in the 2D stochastic problem described in section 4.2 is assumed to follow a Gaussian distribution. The mean normalized wind speed is set to 0.03 with a standard deviation of 0.001 . The blade attachment position has a mean value of 0.25 chords and a standard deviation of 0.01 chords. The objective function is again taken to be the mean power coefficient over the parameter range. Figure 15 shows the final stochastic grid obtained after adaptive sampling colored by the contours of power coefficient. As expected, the sampling procedure has adapted around the center of the stochastic space to minimize errors with high probablity. Figure 16 compares the predicted functional and the error estimate using adaptive and uniform sampling. The effectiveness of adaptive sampling is clearly evident as the error is reduced more rapidly and the adjoint-corrected function reaches a converged value well before the uniformly sampled case.

\section{Summary}

A discrete adjoint solver is developed and used to enable efficient uncertainty quantification in compressible unsteady aerodynamic problems. Specifically, numerical error estimates of functionals arising from approximations of the governing equations in the spatial (via upwind finite volume methods), temporal (time-spectral method) and stochastic (simplex collocation) domains are derived. The effectiveness of the error estimation strategy is verified 


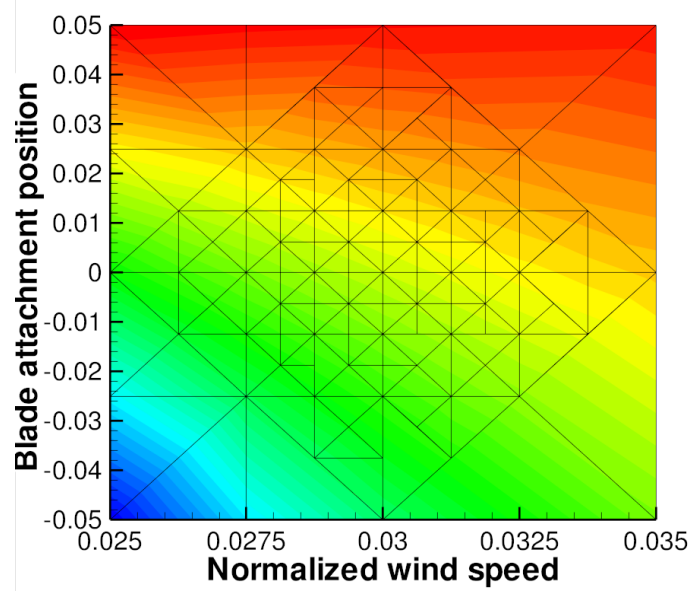

Figure 15: Final stochastic grid with adaptive sampling.

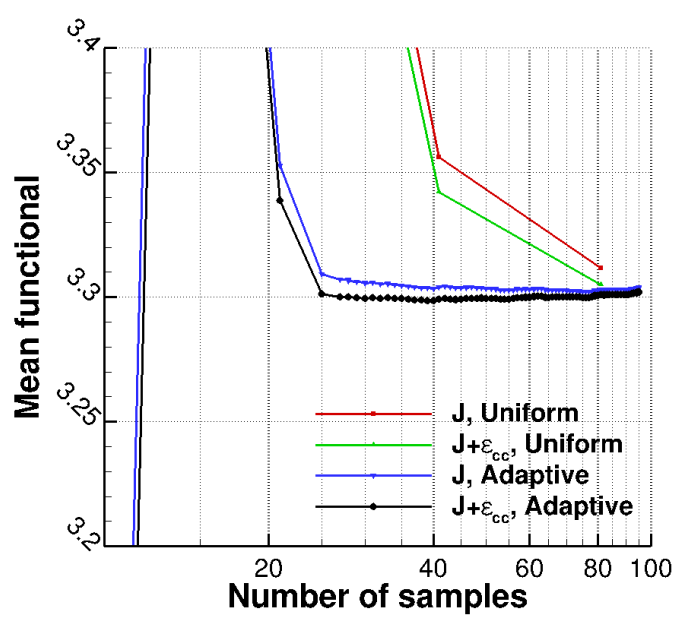

(a) Functional

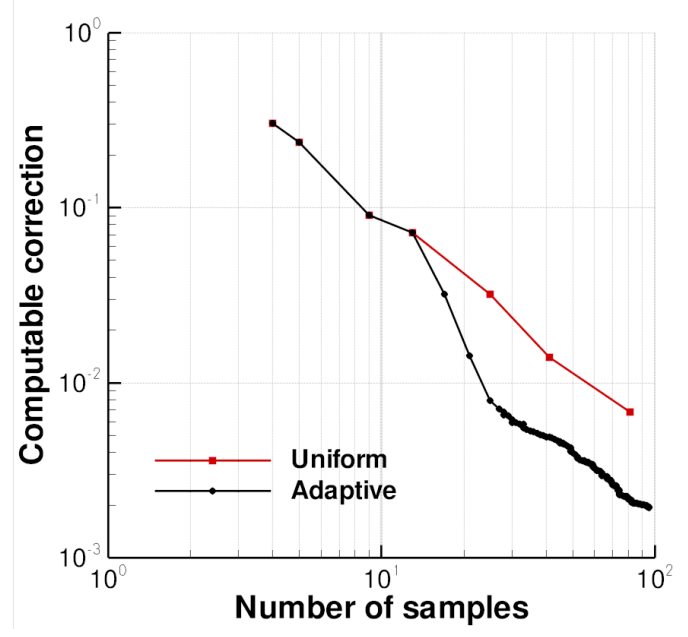

(b) Error

Figure 16: Stochastic error estimation results.

in a modular fashion on steady and unsteady problems. Spatial and stochastic error estimates demonstrate accurate recovery of the error and provide an accurate estimate of the objective functional. The accuracy of the temporal error estimate obtained using spectral interpolation of both flow and adjoint solution significantly under-predict the true error. A newly proposed reconstruction strategy of the adjoint solution in the temporal domain considerably improves the temporal error estimates. As a demonstration, the framework is applied to a vertical axis wind turbine simulation with two uncertain parameters and a budget of the various error sources is computed. The ability to use stochastic error estimates as a basis for adaptive sampling is also demonstrated.

The present work represents progress towards achieving automatic error control in unsteady flows involving ran- 
dom parameters. Ultimately, adaptation indicators will be used to drive the selection of additional stochastic sampling points and refinement of the time-spectral steps and the spatial mesh, simultaneously.

\section{Acknowledgments}

The first author was supported in part by DoE grant DE-FG02-10ER26026. The authors are thankful to Dr. Praveen Chandrashekar for his help with stochastic collocation and Prof. Juan Alonso for fruitful discussions on the topic.

\section{References}

[1] Babuska, I., and Miller, A., "The Post Processing approach in the Finite Element Method, Part 1: Calculation of Stresses, Displacements and Other High Order Derivatives of Displacements," International Journal of Numerical Methods in Engineering, Vol. 20, (6), pp. 1085-1109, 1984.

[2] Baker, T., "Mesh Adaptation Strategies for Problems in Fluid Dynamics," Finite Elements in Analysis and Design, Vol. 25, (3-4), pp. 243-273, 1997.

[3] Giles, M., Larson, M., Levenstam, J., and Suli, E., "Adaptive Error Control for Finite Element Approximations of the Lift and Drag Coefficients in Viscous flow," Technical Report NA-76/06, Oxford University Computing Laboratory, 1997.

[4] Pierce, N., and Giles, M., "Adjoint and Defect Error Bounding and Correction for Functional Estimates," Journal of Computational Physics, Vol. 200, (2), pp. 769-794, 2004.

[5] Venditti, D., and Darmofal, D., "Anisotropic Grid Adaptation for Functional Outputs: Application to Two-dimensional Viscous Flows," Journal of Computational Physics, Vol. 187, (1), pp. 22-46, 2003.

[6] Mani, K., and Mavriplis, D. J., "Error estimation and adaptation for functional outputs in time-dependent flow problems," Journal of Computational Physics, Vol. 229,(2), pp. 415-440, 2010.

[7] Flynt, B., and Mavriplis, D. J., "Optimal Error Control using Discrete Adjoint Error Estimates in Unsteady Flow Problems,” AIAA Paper 20141434, 52nd AIAA Aerospace Sciences Meeting, National Harbor, MD, January 13-17, 2014.

[8] Fidkowski, K., and Darmofal, D., “ Review of Output-Based Error Estimation and Mesh Adaptation in Computational Fluid Dynamics,” AIAA Journal, Vol. 49, (4), pp. 673-694, 2011.

[9] Duraisamy, K., Alonso, J., Palacios, F., and Chandrasekhar, P., "Error Estimation for High Speed Flows Using Continuous and Discrete Adjoints," AIAA Paper 2010-128, 48th Aerospace Sciences Meeting, Orlando, FL, January 4-7, 2010.

[10] Witteveen, J., Duraisamy, K., and Iaccarino, G., “Uncertainty Quantification and Error Estimation in Scramjet Simulation,” AIAA Paper 20112283, 17th AIAA International Space Planes and Hypersonic Systems and Technology Conference, San Francisco, CA, April 11-14, 2011.

[11] Duraisamy, K., and Alonso, J., “Adjoint Based Techniques for Uncertainty Quantification in Turbulent Flows with Combustion,” AIAA Paper 2012-2711, 30th AIAA Applied Aerodynamics Conference, New Orleans, LA, June 25-28, 2012.

[12] Duraisamy, K., and Chandrashekar, P. "Goal Oriented Uncertainty Propagation Using Stochastic Adjoints," Computers and Fluids, Vol. 66, (15), pp. 10-20, 2012.

[13] Palacios, F., Duraisamy, K., Alonso, J. J., and Zuazua, E., "Robust Grid Adaptation for Efficient Uncertainty Quantification," AIAA Journal, Vol. 50, (7), pp. 1538-1546, 2012. 
[14] Gopinath, A., and Jameson, A., "Time spectral method for periodic unsteady computations over two- and three- dimensional bodies," AIAA Paper 2005-1220, 43rd AIAA Aerospace Sciences Meeting and Exhibit, Reno, NV, January 2005.

[15] van der Weide, E., Gopinath, A., and Jameson, A., "Turbomachinery applications with the time spectral method," AIAA Paper 2005-4905, 17th AIAA Computational Fluid Dynamics Conference, Toronto, Canada, June 2005.

[16] Hall, K., Thomas, J., and Clark, W., "Computation of unsteady nonlinear flows in cascades using a harmonic balance technique," AIAA Journal Vol. 40 (5) pp. 879-886, 2002.

[17] Witteveen J., Loeven A., and Bijl, H., "An adaptive stochastic finite elements approach based on NewtonCotes quadrature in simplex elements," Computers and Fluids, Vol. 38, (6), pp. 1270-1288, 2009.

[18] Van Leer, B., “Towards the Ultimate Conservative Difference Scheme V. A Second-Order Sequel To Godunovs Method,” Journal of Computational Physics, Vol. 135, (2), 1997, pp. 229-248.

[19] Roe, P., “Approximate Riemann Solvers, Parameter Vectors and Difference Schemes," Journal of Computational Physics, Vol. 135, (2), pp. 250-258, 1997.

[20] Koren, B., "Multigrid and Defect Correction for the Steady Navier-Stokes Equations," Proceedings of the 11th International Conference on Numerical Methods in Fluid Dynamics, Willamsburg, VA, June 1988.

[21] Jameson, A., "Time Dependent Calculations Using Multigrid, with Applications to Unsteady Flows Past Airfoils and Wings," AIAA Paper 911596, 10th AIAA Computational Fluid Dynamics Conference, Honolulu, HI, June 1991.

[22] Buelow, P. E., Schwer, D. A., Feng, J., Merkle, C., and Choi, D., “A preconditioned dual-time, diagonalized ADI scheme for unsteady computations, AIAA paper 1997-2101, 1997.

[23] Pulliam, T., and Chaussee, D., “A Diagonal Form of an Implicit Approximate Factorization Algorithm,” Journal of Computational Physics, Vol. 39, (2), 1981, pp. 347-363.

[24] Giles, M., Duta, M., Muller, J., and Pierce, N., “Algorithm developments for discrete adjoint methods,” AIAA Journal, Vol. 41, (2), pp. 198$205,2003$.

[25] Mavriplis, D., "Discrete adjoint-based approach for optimization problems on three-dimensional unstructured meshes," AIAA journal, Vol. 45 (4), pp. 741-750, 2007.

[26] Nielsen, E., and Kleb, W., "Efficient construction of discrete adjoint operators on unstructured grids using complex variables," AIAA journal, Vol. 44 (4) pp. 827-836, 2006. APA

[27] Duraisamy, K., Alonso, J., Palacios, F., and Chandrasekhar, P., “Adjoint Based Error Estimation for High Speed Flow Computations," 48th Aerospace Sciences Meeting, Orlando, Florida, Jan, 2010.

[28] Reuther, J. J., Jameson, A., Alonso, J. J., Rimlinger, M. J., and Saunders, D., "Constrained Multipoint Aerodynamic Shape Optimization Using an Adjoint Formulation and Parallel Computers, part 1,” Journal of Aircraft, 36(1), pp. 5160, 1999.

[29] Reuther, J. J., Jameson, A., Alonso, J. J., Rimlinger, M. J., and Saunders, D., “Constrained Multipoint Aerodynamic Shape Optimization Using an Adjoint Formulation and Parallel Computers, part 2," Journal of Aircraft, 36(1), pp. 6174, 1999.

[30] Zymaris A.S., Papadimitriou D.I., Giannakoglou K.C., Othmer C., "Continuous Adjoint Approach to the Spalart-Allmaras Turbulence Model for Incompressible Flows," Computers and Fluids, 38, pp: 1528-1538, 2009.

[31] Hascoët, L., and Pascual, V., “The Tapenade Automatic Differentiation tool: principles, model, and specification,” Research report 7957, INRIA, 2012. http://hal.inria.fr/hal-00695839

[32] Najm, H., "Uncertainty Quantification and Polynomial Chaos Techniques in Computational Fluid Dynamics," Annual Review of Fluid Mechanics Vol. 41, pp. 35-52, 2009.

[33] Dunavant, D., "High degree efficient symmetrical gaussian quadrature rules for the triangle," International Journal for Numerical Methods 
in Engineering, Vol. 21, (6), pp. 1129-1148, 1985.

[34] Oler, J., Strickland, J., Im, B., and Graham, G., "Dynamic Stall Regulation of the Darrieus Turbine," Contractor Report, SAND83-7029, 1983.

[35] Spalart, P., and Allmaras, S., “A One-equation Turbulence Model for Aerodynamic Flows,” AIAA Paper 92-0439, 30th AIAA Aerospace Sciences Meeting and Exhibit, Reno, NV, January 6-9, 1992. 